\title{
ATUAÇÃO FISIOTERAPÊUTICA NA SOBRECARGA FÍSICA E DOR DE CUIDADORES DE CRIANÇAS COM PARALISIA CEREBRAL
}

\author{
Physical therapy intervention in physical stress and pain in caregivers of children with cerebral \\ palsy
}

\section{Actuación fisioterapéutica en la sobrecarga física y el dolor de cuidadores de niños con parálisis cerebral}

\author{
Soraya Sayuri Braga Nohara \\ Universidade Nove de Julho - UNINOVE - São Paulo (SP) - Brasil
}

Sandra Regina Bonifácio

Universidade Nove de Julho - UNINOVE - São Paulo (SP) - Brasil

Karen Tokuhashi Ribeiro

Universidade Nove de Julho - UNINOVE - São Paulo (SP) - Brasil

Fernanda Varkala Lanuez

Universidade Nove de Julho - UNINOVE - São Paulo (SP) - Brasil

Lucia Castro Lemos

Universidade Nove de Julho - UNINOVE - São Paulo (SP) - Brasil

\section{RESUMO}

Objetivo: Avaliar a atuação fisioterapêutica na sobrecarga física e na dor autorreferida de cuidadores de crianças com paralisia cerebral. Métodos: Estudo transversal e longitudinal, realizado em 2017 com 14 cuidadores de crianças com paralisia cerebral que participaram de atuação fisioterapêutica durante cinco semanas consecutivas simultaneamente ao atendimento das crianças, realizado no setor de Fisioterapia em Neuropediatria de um ambulatório de referência do município de São Paulo, Brasil. Para a coleta dos dados, utilizou-se questionário contendo as variáveis escolaridade, sexo, ocupação atual, renda familiar, quantidade de filhos, divisão dos cuidados com outrem, sobrecarga de cuidado, intensidade e localização da dor. Também se utilizou a Avaliação da Sobrecarga do Cuidador e a Escala Visual Analógica (EVA). Resultados: Dos participantes, $12(85,7 \%)$ eram do sexo feminino e $10(71,4 \%)$ não tinham vínculo empregatício. A sobrecarga moderada e baixa manteve a mesma proporção em metade da amostra $(n=7 ; 50 \%)$ após a atividade fisioterapêutica. A relação entre a presença de dor e sobrecarga moderada continuou presente em $62,5 \%(\mathrm{n}=5)$, porém a dor pela escala visual analógica diminuiu, pontuando inicialmente entre 1, como a menor nota, e 10, a maior nota. Após a fisioterapia, a pontuação oscilou entre 0 e 4. Conclusão: A continuidade da prestação do cuidado não cessa e, desse modo, não foi encontrada diferença entre a sobrecarga. Entretanto, após a atuação fisioterapêutica e as orientações recebidas quanto ao posicionamento do cuidador ao manejar a criança, observou-se diminuição das queixas de dores musculoesqueléticas autorreferidas em membros superiores dos cuidadores investigados.

Descritores: Cuidadores; Paralisia Cerebral; Qualidade de Vida; Fisioterapia; Promoção da Saúde.

\section{ABSTRACT}

Objective: To assess physical therapy intervention in self-reported physical stress and pain in caregivers of children with cerebral palsy. Methods: Longitudinal cross-sectional study carried out in 2017 with 14 caregivers of children with cerebral palsy who participated in physical therapy intervention during five consecutive weeks while children were served in the Department of Neuropediatric Physical Therapy of a reference outpatient clinic in the municipality of São Paulo, Brazil. Data were collected using a questionnaire addressing gender, current occupation, household income, number of children, shared caregiving, caregiver overload, and intensity and localization of pain. The Caregiver Burden Interview and the Visual Analog Scale (VAS) were also used. Results: Of the participants, 12 (85.7\%) were women and 10 $(71.4 \%)$ had no employment relationships. Moderate and low overload were found in the same proportion in half of the sample ( $n=7 ; 50 \%)$ after the physical therapy intervention. The relationship between presence of pain and moderate overload remained present in $62 \%(n=5)$ of the participants, but the pain in the visual analog scale decreased, with scores ranging 1 - the lowest - and 10 - the highest. After physical therapy, the score ranged 0 to 4. Conclusion: Caregiving is a nonstop activity; therefore, there were no differences in overload. However, after physical therapy and guidance regarding caregivers' posture while handling the child, there was a decrease in self-reported complaints of musculoskeletal pain in the upper limb of the caregivers analyzed.

Descriptors: Caregivers; Cerebral Palsy; Quality of Life; Physical Therapy Specialty; Health Promotion. 


\section{RESUMEN}

Objetivo: Evaluar la actuación de la fisioterapia en la sobrecarga física y en el dolor auto referido de cuidadores de niños con parálisis cerebral. Métodos: Estudio transversal y longitudinal realizado en 2017 con 14 cuidadores de niños con parálisis cerebral que participan de la actuación de la fisioterapia durante cinco semanas seguidas en las consultas de los niños realizado en el sector de Fisioterapia en Neuropediatria de un ambulatorio de referencia del municipio de São Paulo, Brasil. Para la recogida de datos se utilizó un cuestionario con las variables escolaridad, sexo, ocupación actual, renta familiar, cantidad de hijos, división de los cuidados con otras personas, sobrecarga de cuidado, intensidad y localización del dolor. También se utilizó la Evaluación de la Sobrecarga del Cuidador y la Escala Visual Analógica (EVA). Resultados: De entre los participantes, 12 (85,7\%) eran del sexo femenino y $10(71,4 \%)$ no tenían vinculo de empleo. La sobrecarga moderada y baja se mantuvo en la misma proporción en la mitad de la muestra (n=7; 50\%) después de la actividad de fisioterapia. La relación entre la presencia de dolor y la sobrecarga moderada continuó presente en el 62,5\% $(n=5)$, sin embargo, el dolor disminuyó a través de la escala visual analógica con la puntuación inicial de 1 como la menor nota y 10 la mayor. La puntuación varió entre 0 y 4 después de la fisioterapia. Conclusión: La continuidad de la asistencia del cuidado no termina y, de ese modo, no ha sido encontrada diferencia entre la sobrecarga. Sin embargo, después de la actuación de la fisioterapia y las orientaciones recibidas sobre el posicionamiento del cuidador al cuidar del niño se observó la disminución de las quejas de dolores musculoesqueléticas auto referidas en los miembros superiores de los cuidadores investigados.

Descriptores: Cuidadores; Parálisis Cerebral; Calidad de Vida; Fisioterapia; Promoción de la Salud.

\section{INTRODUÇÃO}

A Paralisia Cerebral (PC) é uma disfunção com predominância sensório-motora que envolve distúrbios no tônus muscular, na postura e na movimentação involuntária. As necessidades apresentadas pelas crianças com PC fazem surgir um novo personagem dentro do ambiente familiar, o cuidador formal ou informal, sendo, na maioria das vezes, a mãe da criança ${ }^{(1,2)}$.

O cuidador se caracteriza como a pessoa responsável por cuidar de um paciente que apresente alguma necessidade especial ou condição de vida que o impossibilite de realizar atividades básicas do cotidiano. O cuidador informal pode ser um familiar ou amigo, alguém que exerce a responsabilidade de cuidar sem remuneração, e o cuidador formal é o profissional habilitado que assume o exercício de cuidar e recebe salário para tal ${ }^{(3)}$.

$\mathrm{O}$ papel do cuidador consiste em assumir a responsabilidade de atender às necessidades básicas da pessoa cuidada e dar suporte visando à melhora da saúde. A intensidade das demandas de cuidados às crianças com necessidades especiais, especificamente com lesão cerebral, torna o cuidado mais complexo e constitui um desafio diário para o cuidador familiar. O cuidador percebe sentimentos difíceis de manejar, que o abalam emocionalmente, e também experimenta sentimento de insegurança, impotência, culpa e desamparo, que têm como consequência privações e mudanças em sua vida, adotando em primeiro plano as ações a favor das necessidades da criança ${ }^{(4,5)}$.

Desse modo, o ato de cuidar leva repercussões negativas para a vida do cuidador, levando a interferências na saúde física e psicológica de quem cuida ${ }^{(6)}$. O desgaste físico e psicológico do cuidador tem sido associado a prejuízos na qualidade de vida. Estudo realizado com 12 cuidadores de crianças portadoras de PC indicou que sentiam dores no corpo, principalmente na coluna, além de cansaço, dificuldade para dormir, má percepção da saúde, depressão, transtorno de humor, irritabilidade, incertezas quanto ao modo de agir e problemas no convívio social, familiar e profissional ${ }^{(7)}$.

A sobrecarga é uma perturbação resultante do lidar com a dependência física e/ou a incapacidade mental do indivíduo centro da atenção e dos cuidados. Assim, a qualidade de vida e manutenção da saúde é ameaçada em decorrência das responsabilidades como cuidador, apresentando sentimento de impotência, preocupação, cansaço e irritabilidade em muitas situações. E o cuidado com o filho causa um sofrimento às vezes invisível aos profissionais de saúde que estão focados no tratamento da criança com paralisia cerebral, e este sofrimento não reconhecido pode acarretar no adoecimento dos cuidadores, o que pode gerar grande impacto social, alteração na dinâmica familiar e alto custo para o sistema de saúde, sendo necessárias ações de saúde coletiva para promover um suporte de modo que sejam melhor assistidos e promovam o autocuidado ${ }^{(8,9)}$.

A criança com paralisia cerebral necessita de cuidado contínuo, com auxilio ou total dependência para realizar atividades básicas da vida diária, o que acarreta na sobrecarga física do cuidador. O cuidado com o cuidador é um processo complexo que visa melhorar as condições de saúde física, emocional e social. Muito se estuda sobre o impacto da doença na vida do cuidador, mas são raras as ações de promoção à saúde e prevenção de agravos destinadas a eles, seja pela dificuldade de terem outra pessoa para dividir a tarefa de cuidar, seja pelo maior tempo despendido no cuidado da criança.

Sendo assim, o objetivo deste estudo foi avaliar a atuação fisioterapêutica na sobrecarga física e na dor autorreferida de cuidadores de crianças com paralisia cerebral.

\section{MÉTODOS}

Trata-se de estudo transversal e longitudinal, realizado no período de março a abril de 2017, no setor de Fisioterapia em Neuropediatria do Ambulatório Integrado de Saúde da Vila Maria (AIS-VM), situado em São Paulo, São Paulo, Brasil. Foram 
convidados 29 cuidadores, dos quais 14 aceitaram o convite de participar do estudo e atenderam aos critérios de inclusão, os quais eram: serem cuidadores principais e terem interesse em participar do estudo simultaneamente à fisioterapia da criança com paralisia cerebral. E os critérios de exclusão consistiram em usuários e/ou famílias que não fizessem parte do setor de Fisioterapia em Neuropediatria e pessoa que presta cuidados ocasionais à criança com paralisia cerebral. A amostra ficou, então, constituída por 14 cuidadores que concordaram voluntariamente em participar da pesquisa.

A coleta de dados ocorreu através do preenchimento de um questionário contendo variáveis como escolaridade, sexo, ocupação atual, renda familiar, quantidade de filhos, divisão dos cuidados com outrem, sobrecarga de cuidado, intensidade da dor e localização da dor. Para analisar a sobrecarga dos cuidados, utilizou-se a Avaliação da Sobrecarga dos Cuidadores Zarit ${ }^{(10)}$ e empregou-se a Escala Visual Analógica - EVA ${ }^{(11)}$ para avaliar a intensidade das queixas de dores musculoesqueléticas.

A Avaliação da Sobrecarga dos Cuidadores - Zarit( ${ }^{(10)}$ é um instrumento de 22 itens para avaliar a sobrecarga associada à incapacidade funcional e comportamental do paciente e com a situação em casa. Os itens referem áreas de preocupação, tais como: saúde, vida social, vida pessoal, situação financeira, bem-estar e relacionamentos, que medem objetiva e subjetivamente a sobrecarga dos cuidadores. No presente estudo, os itens foram avaliados de acordo com o que ocorreu durante o mês anterior à entrevista. Cada item é pontuado de 0 a 4 , sendo $0=$ Nunca, $1=$ Raramente, $2=$ Algumas vezes, $3=$ Frequentemente, $4=$ Sempre. Somam-se todos os itens, pode variar de 0 a 88. Quanto maior o escore, maior a carga.

A Escala Visual Analógica (EVA) ${ }^{(11)}$ é um instrumento unidimensional para avaliar a intensidade da dor. Trata-se de uma linha com as extremidades numeradas de 0 a 10. Em uma extremidade da linha é marcada "nenhuma dor" e na outra "pior dor imaginável", solicitando-se que o paciente possa avaliar sua dor e marcar na linha a dor no momento.

Após a coleta de dados, os cuidadores foram convidados a participar de um programa de atuação fisioterapêutica em grupo. As atividades tiveram como objetivo a diminuição da sobrecarga do cuidado e da dor autorreferida dos cuidadores através de exercícios de alongamento dos músculos: serrátil anterior, elevador da escápula, trapézio, paravertebrais cervicais, peitoral maior, flexores de coluna cervical, flexores e extensores do punho, paravertebrais lombares, glúteos, isquiostibiais e quadríceps, de modo ativo assistido e ativo (30 segundos). Fizeram-se exercícios respiratórios com padrão diafragmático (3 ciclos de 5 repetições) e fortalecimento isométrico de rombóides, flexores, abdutores e rotadores de ombros, abdome, paravertebrais lombares e quadríceps, e fortalecimento dos músculos posteriores inferiores com faixa elástica da cor verde evoluindo para a azul (3 séries de 10 repetições). Também receberam orientação quanto ao manejo e transferência das crianças para não sobrecarregar sua coluna vertebral.

Os encontros ocorreram duas vezes por semana, com duração de 45 minutos cada, totalizando 10 sessões consecutivas de fisioterapia. Após 5 semanas, os participantes foram reavaliados com os mesmos instrumentos. Os dados sofreram registro em banco de dados e posteriormente realizou-se a análise descritiva.

A presente pesquisa recebeu aprovação do Comitê de Ética em Pesquisa da UNINOVE (Parecer nº 1912173).

\section{RESULTADOS}

Dentre a amostra constituída de 14 cuidadores, 12 (85,7\%) eram do sexo feminino, com idade média de 38 anos, sendo que $11(78,6 \%)$ eram mães da criança e outros $3(21,4 \%)$ tinham outro parentesco.

A renda familiar ficou concentrada em 1 (um) salário mínimo, como demonstrado na Tabela I. Dentre os cuidadores, 10 $(71,4 \%)$ não tinham vínculo empregatício para contribuir com o sustento da família.

Tabela I - Distribuição do número e porcentagem dos cuidadores de crianças com paralisia cerebral segundo as variáveis socioeconômicas. São Paulo, São Paulo, 2017 (n=14).

\begin{tabular}{|c|c|c|}
\hline Variável & $\mathbf{n}$ & $(\%)$ \\
\hline \multicolumn{3}{|l|}{ Escolaridade } \\
\hline Fundamental incompleto & 2 & 14,3 \\
\hline Médio completo/ incompleto & 10 & 71,4 \\
\hline Superior completo/ incompleto & 2 & 14,3 \\
\hline \multicolumn{3}{|l|}{ Renda Familiar } \\
\hline 1 salário mínimo & 9 & 64,3 \\
\hline 2 salários mínimos & 3 & 21,4 \\
\hline 3 salários mínimos ou mais & 2 & 14,3 \\
\hline \multicolumn{3}{|l|}{ Quantidade de filhos } \\
\hline 1 & 5 & 35,7 \\
\hline 2 & 5 & 35,7 \\
\hline 3 ou mais & 4 & 28,6 \\
\hline \multicolumn{3}{|l|}{ Trabalha atualmente } \\
\hline Sim & 4 & 28,6 \\
\hline Não & 10 & 71,4 \\
\hline Total & 14 & 100 \\
\hline
\end{tabular}


Na Avaliação de Sobrecarga do Cuidador (Zarit), constatou-se que, antes da fisioterapia, 7 (50\%) apresentaram sobrecarga moderada e 7 (50\%), baixa sobrecarga. Os cuidadores que se queixaram de dor osteomuscular foram 9 (64,3\%), e a coluna lombar representou o local de maior frequência de dor 5 (35,8\%) (Tabela II).

No presente estudo, os cuidadores eram os principais responsáveis pelos cuidados das crianças; sem tempo para descanso, autocuidado e lazer; relacionando o nível de sobrecarga com a presença da dor. Sugere-se, como mostram os resultados da Tabela II, que, na presença da dor, a sobrecarga moderada não se altera, pois 5 cuidadores apresentaram dor e sobrecarga moderada antes e depois da atuação fisioterapêutica. Apenas um (1) cuidador diminuiu a dor e a sobrecarga foi baixa, sugerindo associação entre a queixa de presença da dor musculoesquelética e uma maior sobrecarga.

Tabela II - Nível de sobrecarga com a presença de relato de dor musculoesquelética antes e após proposta de atuação fisioterápica. São Paulo, São Paulo, 2017. (n=14).

\begin{tabular}{|c|c|c|c|c|c|c|}
\hline \multirow[b]{3}{*}{ Dor } & \multicolumn{5}{|c|}{ Sobrecarga } & \multirow[b]{2}{*}{ Total } \\
\hline & \multicolumn{2}{|c|}{ Antes } & \multirow{2}{*}{$\begin{array}{l}\text { Total } \\
\text { n (\%) }\end{array}$} & \multicolumn{2}{|c|}{ Depois } & \\
\hline & $\begin{array}{l}\text { Baixo } \\
\text { n (\%) }\end{array}$ & $\begin{array}{c}\text { Moderado } \\
\text { n (\%) }\end{array}$ & & $\begin{array}{l}\text { Baixo } \\
\text { n (\%) }\end{array}$ & $\begin{array}{c}\text { Moderado } \\
\text { n (\%) }\end{array}$ & n (\%) \\
\hline Não & $3(60,0)$ & $2(40,0)$ & $5(100)$ & $4(66,7)$ & $2(33,3)$ & $6(100)$ \\
\hline Sim & $4(44,4)$ & $5(55,6)$ & $9(100)$ & $3(37,5)$ & $5(62,5)$ & $8(100)$ \\
\hline Total & $7(50,0)$ & $7(50)$ & $14(100)$ & $7(50,0)$ & $7(50,0)$ & $14(100)$ \\
\hline
\end{tabular}

$\mathrm{n}=$ número de indivíduos; \%= percentual.

Dentre os 9 (64,3\%) que referiram dor, a prevalência antes da atuação fisioterapêutica foi a coluna lombar (35,8\%), e este valor permaneceu inalterado após. Porém a dor em membros superiores, que antes da intervenção era queixa de 4 (28,5\%), diminuiu para $3(21,4 \%)$. Obteve-se uma diminuição de $9(64,3 \%)$ para $8(57,2 \%)$ no número de cuidadores que referiram dor após a atuação fisioterapêutica.

Em relação ao EVA, antes da atuação fisioterapêutica, a queixa de dor recebeu pontuação 1 para a menor nota de dor referida e a maior nota recebeu 10, sendo que, após a atuação fisioterapêutica, nenhuma dor foi referida e a maior pontuação foi 4. Estes resultados evidenciam a melhora da percepção da queixa de dor autorreferida pelos participantes do presente estudo.

\section{DISCUSSÃO}

Apesar de toda a família sentir a doença da criança, é o cuidador quem assume o cuidado do paciente na assistência física, emocional e até financeira. É frequente o cuidado ser efetuado por cuidadores do sexo feminino, pois na sociedade brasileira a mulher é a responsável pelo cuidado da casa e dos filhos, implicando em perdas de seu tempo livre e cuidados com a sua própria saúde, repercutindo em sua vida social. No passado, as mulheres não realizavam funções fora de casa, propiciando maior disponibilidade para o cuidado com a família ${ }^{(4,12)}$.

A baixa renda familiar da amostra do presente estudo corrobora achados de outros trabalhos ${ }^{(2,13)}$ uma vez que cuidar de crianças com incapacidade aumenta a demanda de recursos e pode influenciar na sobrecarga dos cuidadores em famílias com condições socioeconômicas menos favorecidas. A maioria dos cuidadores do presente estudo não trabalhava no momento, assim como averiguado em estudos em que 91,6\% e 70,8\% dos cuidadores, respectivamente, não possuíam ocupação ou renda fora do lar, o que pode contribuir para a sobrecarga do cuidado ${ }^{(14,15)}$.

Como consequência do cuidado, que é uma tarefa complexa e que exige tempo e dedicação, os cuidadores acabam deixando de lado seu bem-estar e vivem em função da criança ${ }^{(14,16)}$. A sobrecarga envolve não somente aspectos físicos, mas também psicológicos, familiares, financeiros e sociais. Em várias situações o cuidador principal sofre perdas financeiras, negligências e abandono por parte dos demais familiares, ficando sobrecarregado com a tarefa de cuidar ao assumir a função ${ }^{(17)}$.

O estudo em questão limitou-se à sobrecarga física, pois o conjunto diário de esforços empregues, que geralmente excedem suas capacidades físicas, associado à falta de tempo e de descanso, levam à negligência da sua própria saúde, o que pode ter influenciado no resultado, o qual mostrou que a sobrecarga moderada se manteve na mesma proporção e apenas 1 cuidador diminuiu a sobrecarga, de moderada para baixa.

Os resultados do atual estudo sugerem associação entre dor e sobrecarga moderada, pois quanto maior a sobrecarga maior a chance de queixas de dores e de sua relação com a prestação do cuidado ${ }^{(18)}$. O acúmulo de responsabilidades e tarefas que o cuidador assume, além da dedicação permanente, ocasiona esforço físico contínuo, necessitando de força muscular e postura adequadas para realizar as diversas necessidades das crianças, como auxílio no banho, transferências e ajudar na locomoção, tornando as atividades da vida diária das crianças mais complicadas de acordo com o crescimento e peso delas ${ }^{(6,9)}$.

As crianças com PC apresentam distúrbios de postura e movimento que levam a uma perda motora e dificuldades para realizar as atividades de vida diária. Por consequência, uma dependência funcional que acarreta uma sobrecarga física, 
identificada no estudo em que avaliaram 30 mães e encontraram queixa álgica em $86,6 \%$ dos casos devido ao manejo das crianças nesses cuidados diários ${ }^{(19,20)}$.

Em um estudo realizado na cidade de Salvador, Bahia ${ }^{(21)}$, os cuidadores pesquisados pontuaram a sobrecarga musculoesquelética como um componente essencial, justificando, desse modo, a não diminuição na frequência da dor na região da coluna lombar, posto que as atividades realizadas para o cuidado da criança demandam grande força muscular pela necessidade das transferências e locomoções diárias, somadas ao próprio peso corporal, como as transferências posturais da criança de uma cama para uma cadeira de rodas, o que pode ser explicado pela biomecânica corporal imposta por cargas excessivas nas atividades diárias. Além de carregarem o próprio peso, têm que vencer a carga imposta pelo peso da criança que carregam para conseguir realizá-las ${ }^{(21)}$. A frequência de dor está relacionada ao peso da criança que durante os cuidados diários sobrecarrega a coluna vertebral ${ }^{(19)}$. Resultados semelhantes foram encontrados no estudo em que os cuidadores referiram dor lombar em uma frequência de $28 \%^{(22,23)}$.

A diminuição de dor autorreferida dos membros superiores confirma os resultados do estudo em que os exercícios localizados nessa região resultaram em uma redução significativa da dor em um grupo de 39 mulheres ao longo de um período de 7 semanas ${ }^{(24)}$.

Há poucas pesquisas sobre intervenção na sobrecarga do cuidado e dor nos cuidadores na literatura. Há um estudo com 12 cuidadores, em que foram realizadas somente orientações fisioterapêuticas e reavaliados após 5 semanas, havendo uma pequena redução da dor, mas não na sobrecarga do cuidador ${ }^{(25)}$, analisadas através do Questionário de Avaliação da Sobrecarga do Cuidador Informal.

A dificuldade não está somente no cuidar, mas também em abdicar de necessidades próprias em função do outro, levando a uma diminuição do tempo de cuidado consigo e das atividades de lazer, uma vez que o recebimento da notícia da doença da criança em um primeiro momento é inesperado e todos se deparam com o desconhecido, ocorrendo diversas mudanças na estrutura familiar. A criança terá que ter um acompanhamento para realizar por ela ou auxiliá-la em funções, como na alimentação, vestimenta, higiene pessoal, locomoção, entre outras ${ }^{(26)}$. Esse papel é assumido pelo cuidador, os quais, pela falta de apoio familiar, abandono de emprego e a sobrecarga de atividades no lar, podem repercutir na sua qualidade de vida, ocorrendo a sobrecarga do cuidador ${ }^{(27)}$.

O presente estudo confirma o que a literatura indica: a sobrecarga do cuidado incide sobre a figura feminina, no caso a mãe da criança, sendo mais exposta do que outros familiares, pois, nesta situação, está mais envolvida nos cuidados, requerendo que ela se coloque em segundo plano e adote um papel suplementar àqueles já desempenhados. Essa motivação para o cuidado é uma opção pessoal e está associada a sentimentos de amor e respeito, especialmente quando há um forte vínculo entre a mãe e a criança, pois sentem-se gratificadas ao observar que conseguem se reestruturar mesmo em frente a dificuldades impostas no dia a $\operatorname{dia}^{(2,27,28)}$.

O atual estudo teve como limitação a não adesão dos cuidadores em participar do estudo, pois os horários das atuações fisioterapêuticas aconteciam simultaneamente ao atendimento das crianças na fisioterapia, resultando em perda amostral. Um outro ponto importante refere-se ao número de atendimentos, que pode ter limitado a melhora dos pacientes frente a sobrecarga do cuidado. Mesmo assim, o presente estudo mostra a importância de cuidar do cuidador, que assim como a pessoa cuidada, também é usuário do serviço de saúde e requer atenção específica, inclusive de caráter preventivo, visando alternativas de cuidar-se sem abster-se do papel assumido e a promoção da saúde e da qualidade de vida, com melhora nas suas condições físicas, sociais e emocionais ${ }^{(8)}$.

Uma das propostas do atual estudo foi otimizar o tempo em que os cuidadores aguardavam no ambulatório o atendimento das crianças, procurando proporcionar o autocuidado para a promoção da saúde. Desse modo, há a necessidade de estudos futuros direcionados aos cuidadores de crianças com paralisia cerebral, tendo em vista a dificuldade do cuidado próprio devido à demanda despendida pela criança, além da elaboração de propostas para minimizar a demanda física e emocional inerente ao cuidar, prevenindo possíveis adoecimentos aos cuidadores, especificando dias pontuais para o atendimento fisioterapêutico e maior número de atendimentos, pois possibilitará, desse modo, atuação fisioterapêutica mais efetiva na mudança do perfil de saúde dessa população.

\section{CONCLUSÃO}

A continuidade da prestação do cuidado não cessa e, desse modo, não foi encontrada diferença entre a sobrecarga. Entretanto, após a atuação fisioterapêutica e as orientações recebidas quanto ao posicionamento do cuidador ao manejar a criança, observouse diminuição das queixas de dores musculoesqueléticas autorreferidas em membros superiores dos cuidadores investigados.

\section{REFERÊNCIAS}

1. Oliveira FL, Dounis AB. As alterações na dinâmica familiar diante do diagnóstico da criança com Paralisia Cerebral: Estado da Arte. Rev Psicol (Fortaleza). 2012;3(1):18-27. 
2. Barbosa DC, Souza FGM, Silva ACO, Silva IR, Silva TP, Paiva MCM. Sobrecarga do cuidado materno à criança com condição crônica. Cogitare Enferm. 2012;17(3):492-7.

3. Pereira RA, Santos EB, Fhon JRS, Marques S, Rodrigues RAP. Sobrecarga dos cuidadores de idosos com acidente vascular cerebral. Rev Esc Enferm USP. 2013;47(1):185-92.

4. Dantas MSA, Pontes JF, Assis WD, Collet N. Facilidades e dificuldades da família no cuidado à criança com paralisia cerebral. Rev Gaúch Enferm. 2012; 33(3):73-80.

5. Oliveira APP, Caldana RHL. As repercussões do cuidado na vida do cuidador familiar do idoso com demência de Alzheimer. Saúde Soc. 2012;21(3):675-85.

6. Souza LR, Hanus JS, Libera LBD, Silva VM, Mangilli EM, Simões PW, et al. Sobrecarga no cuidado, estresse e impacto na qualidade de vida de cuidadores domiciliares assistidos na atenção básica. Cad Saúde Colet (Rio J). 2015;23(2):140-9.

7. Moretti GS, Moreira KA, Pereira SR. Qualidade de vida dos cuidadores de crianças com paralisia cerebral da Amazônia sul ocidental. Rev Bras Promoção Saúde. 2012;25(1):30-6

8. Borges CL, Cunha JP, Silva AA, Rocha VA, Freitas MC. Cuidando do cuidador: intervenções para o autocuidado. Rev Enferm UFPE, 2015;9(4):7474-81.

9. Geere JL, Gona J, Omondi FO, Kifalu MK, Newton CR, Hartley S. Caring for children with physical disability in Kenya: potential links between caregiving and carers' physical health. Child Care Health Dev. 2013;39(3):381-92.

10. Krön CA, Simões MLG. Avaliação de sobrecarga em cuidadores: um estudo de revisão sobre a escala Zarit Burden Interview. In: Anais do II Encontro de Iniciação em Desenvolvimento Tecnológico e Inovação, 2013 Set 24 e 25 [acesso em 2017 Abr 15]. Disponível em: https://www.puc-campinas.edu.br/websist/Rep/Sic08/Resumo/201388_223938_364825947_ resC $\% 20 \mathrm{~K} . p d f$

11. Fortunato JG, Furtado MS, Hirabae LFA, Oliveira JA. Escalas de dor no paciente crítico: uma revisão integrativa. Rev Hospital Universitário Pedro Ernesto. 2013;12(3):110-7

12. Gratão ACM, Talmelli LFS, Figueiredo LC, Rosset I, Freitas CP, Rodrigues RAP. Dependência funcional de idosos e a sobrecarga do cuidador. Rev Esc Enferm USP. 2013;47(1):137-44.

13. Ribeiro M, Ferreira M, Porto CC, Vandenberghe L. Estresse parental em famílias de crianças com paralisia cerebral: revisão integrativa. Ciênc Saúde Coletiva. 2013;18(6):1705-15.

14. Gondim KDM, Carvalho ZMDF. Sentimentos das mães de crianças com paralisia cerebral à luz da teoria de Mishel. Esc Anna Nery Rev Enferm. 2012;16(1):11-6.

15. Mello R, Ichisato SMT, Marcon SS. Percepção da família quanto à doença e ao cuidado fisioterapêutico de pessoas com paralisia cerebral. Rev Bras Enferm. 2012;65(1):104-9.

16. Fuhrmann AC, Bierhals CCBK, Santos NO, Paskulin LMG. Association between the functional capacity of dependent elderly people and the burden of family caregivers. Rev Gaúch Enferm. 2015;36(1):14-20

17. Baptista BO, Beuter M, Girardon-Perlini NMO, Brondani CM, Budó MLD, Santos NO. A sobrecarga do familiar cuidador no âmbito domiciliar: uma revisão integrativa da literatura. Rev Gaúch Enferm. 2012;33(1):147-56.

18. Santos WJ, Albuquerque PC, Fittipaldi EOS. Análise do perfil e sobrecarga de cuidadores de três Unidades de Saúde da Família do Recife/PE. Fisioter Bras. 201617(5):464-71.

19. Ferreira MC, Naccio BLD, Otsuka MYC, Barbosa AM, Corrêa PFL, Gardenghi G. Avaliação do índice de sobrecarga de cuidadores primários de crianças com paralisia cerebral e sua relação com a qualidade de vida e aspectos socioeconômicos. Acta Fisiátrica. 2015;22(1):9-13

20. Almeida LMS, Vasconcelos RS, Sales RP, Matos WLR, Viana SMNR, Andrade EA, et al. Prevalência de algias da coluna vertebral em mães de crianças com paralisia cerebral não deambulantes. Rev Saúde Pública Santa Catarina. 2012;5(1):2837.

21. Almeida MS, Conceição TMA. Prevalência de sintomas álgicos, sobrecarga e qualidade de vida de cuidadores de crianças com paralisia cerebral. Rev Pesquisa Fisioterapia. 2013;3(1):36-49.

22. Yeung SS. Factors contributing to work related low back pain among personal care workers in old age. Work. 2012;41(Suppl 1):1873-83. 
23. Almeida KM, Fonseca BM, Gomes AA, Oliveira MX. Fatores que influenciam a qualidade de vida de cuidadores de paralisados cerebrais. Fisioter Mov. 2013;26(2):307-14

24. Rett MT, Mesquita PDJ, Mendonça ARC, Moura DP, Santana JM. A cinesioterapia reduz a dor no membro superior de mulheres submetidas à mastectomia ou quadrantectomia. Rev Dor. 2012;13(3):201-7.

25. Fernandes BCW, Ferreira KCP, Marodin MF, Val MON, Fréz AR. Influência das orientações fisioterapêuticas na qualidade de vida e na sobrecarga de cuidadores. Fisioter Mov. 2013;26(1):151-8.

26. Almeida TCS, Ruedell AM, Nobre JR, Tavares KO. Paralisia Cerebral: Impacto no Cotidiano Familiar. Reva Bras Ciênc Saúde. 2015;19(3):171-8.

27. Baltor MRR, Dupas G. Experiências de famílias de crianças com paralisia cerebral em contexto de vulnerabilidade social. Rev Latinoam Enferm [Internet]. 2013 [acesso em 2017 Mar 18];21(4):[8 telas]. Disponível em: https://www.revistas.usp. br/rlae/article/view/76009/79614

28. Simões CC, Silva L, Santos MR, Misko MD, Bousso RS. A experiência dos pais no cuidado dos filhos com paralisia cerebral. Rev Eletrônica Enferm. 2013;15(1):138-45.

\section{Endereço do primeiro autor:}

Soraya Sayuri Braga Nohara

Universidade Nove de Julho - UNINOVE

Rua Vergueiro, 235/249

Bairro: Liberdade

CEP: 01504-001 - São Paulo - SP - Brasil

E-mail: sora_sayuri@hotmail.com

\section{Endereço para correspondência}

Fernanda Varkala Lanuez

Universidade Nove de Julho - UNINOVE

Rua Vergueiro, 235/249

Bairro: Liberdade

CEP: 01504-001 - São Paulo - SP - Brasil

E-mail: fernandavarkala@uninove.br 